\title{
Genealogia acadêmica dos pesquisadores da área de Ciência da Informação: um estudo sobre os bolsistas de produtividade em pesquisa (PQ-CNPq)
}

\author{
Carlos Alexandre de Oliveira \\ Doutorando; Universidade Federal de Minas Gerais, Belo Horizonte, MG, Brasil; \\ oliveiraca@ufmg.br \\ Marlene Oliveira \\ Doutora; Universidade Federal de Minas Gerais, Belo Horizonte, MG, Brasil; \\ marleneotmelo@gmail.com \\ Thiago Magela Rodrigues Dias \\ Doutor; Centro Federal de Educação Tecnológica de Minas Gerais, Belo Horizonte, MG, Brasil; \\ thiagomagela@gmail.com \\ Belkiz Inez Rezende Costa \\ Mestranda; Universidade Federal de Minas Gerais, Belo Horizonte, MG, Brasil; \\ belkizcosta@gmail.com
}

\begin{abstract}
Resumo: O artigo tem como objetivo investigar a genealogia acadêmica dos pesquisadores bolsistas de produtividade em pesquisa do Conselho Nacional de Desenvolvimento Científico e Tecnológico, do nível 1 (PQ1-CNPq) em Ciência da Informação (CI). Assim, espera-se mapear a propagação do conhecimento através do pesquisador/orientador, além de evidenciar a contribuição dos pesquisadores na formação de recursos humanos para a área. Adota como procedimentos metodológicos: identificação dos bolsistas de produtividade em pesquisa em CI; coleta de dados na Plataforma Lattes e na Plataforma Acácia; análise e apresentação das árvores genealógicas acadêmicas dos pesquisadores. Os resultados obtidos demonstraram que os PQ1-CNPq em CI orientaram 627 pesquisadores, tiveram 1.075 descendentes e linhagem acadêmica de quarta geração. Foram identificados também os ascendentes acadêmicos dos pesquisadores bolsistas, destacando aqueles ancestrais acadêmicos que mais contribuíram para a formação das gerações de pesquisadores da CI brasileira.
\end{abstract}

Palavras-chave: Genealogia acadêmica. Orientação acadêmica. Ciência da Informação. Bolsa de produtividade em pesquisa. Estudos genealógicos.

\section{Introdução}

A divulgação dos resultados de pesquisas em publicações reconhecidas pela comunidade científica, possibilitando a troca de informações e ideias entre os seus membros, é condição necessária para o contínuo progresso e 
desenvolvimento da ciência (Le COADIC, 1996; MEADOWS,1999). O avanço da ciência requer ainda a existência de uma nova geração de pesquisadores disposta e capaz de manter o sistema de produção da ciência em pleno funcionamento (HEINISCH; BUENSTORF, 2018). Nesse aspecto, a orientação, supervisão ou mentoria acadêmica ${ }^{1}$ dá uma importante contribuição ao contínuo progresso da ciência, uma vez que é parte responsável pela formação e evolução de novos pesquisadores e, consequentemente, pela realização de novos estudos em diversas áreas do conhecimento (CORDOVA; FURUKAWA; YAGHI, 2015).

A orientação acadêmica é um processo em que um pesquisador experiente (orientador) instrui um outro pesquisador iniciante (orientando) na realização de atividades de pesquisa e qualificação acadêmica. $\mathrm{O}$ orientador ocupa um papel central nesse processo, contribuindo para a construção coletiva do conhecimento e para formação de novas gerações de pesquisadores (BIANCHETTI; MACHADO, 2012). As contribuições advindas da relação orientador-orientado podem extrapolar no tempo e no espaço por meio da perpetuação da herança intelectual do orientador. Essa pode ser duradoura e continuar a influenciar o pensamento científico nas gerações seguintes de novos pesquisadores.

No âmbito acadêmico/científico, a excelência do pesquisador/docente é avaliada, geralmente, por meio da aplicação de métodos matemáticos e estatísticos para a qualificação da produção científica de um pesquisador (MENA-CHALCO et al., 2014). Essa avaliação considera conceitos relacionados principalmente com as áreas de Bibliometria, Cientometria e suas derivações, a depender do tipo de canal considerado para divulgação (GUEDES; BORSCHIVER, 2005).

Porém, recentemente, pode-se observar diferentes iniciativas, por parte de comunidades acadêmicas específicas, que medem a qualificação de pesquisadores por meio da análise de suas contribuições na formação de recursos humanos. Iniciativas dessa natureza delineiam um campo de estudo que se denomina Genealogia Acadêmica (GA). 
A GA é utilizada para documentar e organizar, através de uma árvore (ou conjuntos de árvores) de genealogia, pesquisadores por meio de suas relações (linhagem) de orientação ou supervisão acadêmica. A análise da GA é constituída de estudos da herança intelectual, operacionalizada pelas relações interdependentes entre os alunos e seus orientadores (SUGIMOTO, 2014). Consiste em um tipo específico de rede social, caracterizada como árvore, e de colaboração científica, uma vez que seus atores são os orientadores e orientandos.

Nesse contexto, pretende-se investigar a genealogia acadêmica dos pesquisadores bolsistas de produtividade em pesquisa do Conselho Nacional de Desenvolvimento Científico e Tecnológico, do nível 1 (PQ1-CNPq) na área de Ciência da Informação (CI). De forma específica, pretende-se: i) identificar os principais ancestrais acadêmicos dessa área; ii) ranquear os bolsistas por taxa de fecundidade e de descendentes; iii) identificar os bolsistas com maior geração ou linhagem acadêmica. Acredita-se que o estudo da genealogia acadêmica dos bolsistas permitirá mapear a propagação do conhecimento dessa área através do pesquisador/orientador, além de evidenciar a contribuição dos pesquisadores na formação de recursos humanos para a CI.

A escolha deste grupo seleto se deve ao fato que o CNPq concede, por meio de edital específico, a bolsa de produtividade em pesquisa para pesquisadores que se destacam em determinadas áreas de conhecimento, valorizando a produção científica, segundo critérios estabelecidos pelos Comitês de Assessoramento do CNPq. As bolsas são distribuídas, segundo alguns autores, aos pesquisadores que constituem a elite da produção científica no país, pois, além de outros critérios, os bolsistas devem dedicar-se ao cumprimento dos propósitos constantes nos projetos de pesquisa, mantendo atividades acadêmicocientíficas, oficialmente vinculadas a instituições de pesquisa e ensino (BUFREM; SILVA; SOBRAL; 2017; CAFÉ et. al., 2011)

As bolsas de produtividade em pesquisa são classificadas em 3 níveis: nível 2 (PQ2), nível 1 (PQ1) e nível sênior (PQ-Sr), em que o nível 2 representa o menor nível de excelência e o nível sênior representa o maior nível de excelência. Nota-se ainda que o nível 1 é subdividido em outros quatro níveis: 
1-D, 1-C, 1-B e 1-A. Neste contexto, a progressão de uma faixa para outra ocorre por avaliação comparativa entre os pesquisadores da área e com base nos dados dos últimos dez anos do pesquisador, de tal modo a demonstrar produção científica regular, liderança científica na área e capacidade de formação contínua de recursos humanos (GUIMARÃES; GRACIO; MATOS, 2014).

O presente trabalho trata-se de uma versão ampliada de um outro (OLIVEIRA; COSTA; OLIVEIRA, 2018) que foi apresentado no $6^{\circ}$ Encontro Brasileiro de Bibliometria e Cientometria ( $\left.6^{\circ} \mathrm{EBBC}\right)$, realizado no Rio de Janeiro, nos dias 17 a 20 de julho de 2018. O trabalho intitulado "Genealogia Acadêmica: um estudo dos pesquisadores da área de Ciência da Informação com bolsas produtividade em pesquisa na modalidade PQ-1 do CNPq" foi apresentado na sessão oral do referido evento. Os trabalhos se diferenciam em seus aspectos teóricos e empíricos. Este apresenta escopo de pesquisa, métodos de extração de dados e análises distintos daquele apresentado no $6^{\circ} \mathrm{EBBC}$.

\section{Genealogia acadêmica}

A GA é o estudo da herança intelectual que é perpetuada por meio de relacionamentos de orientação acadêmica (SUGIMOTO, 2014). As atividades de orientação acadêmica promovem a propagação de conhecimento científico por meio da interação entre orientador-orientado, sendo esse último comumente influenciado pelas características do primeiro. Fornecer os meios para mensurar e analisar essas interações formais de propagação de conhecimento é a função da GA (ROSSI; DAMACENO; MENA-CHALCO, 2018).

A formação de novos cientistas geralmente é representada ao longo dos anos como uma espécie de árvore genealógica acadêmica, sendo muito semelhante à conhecida árvore genealógica. Ela consiste simplesmente em um gráfico direto, no qual os nós representam pesquisadores e as relações indicam que um pesquisador era o orientador de outro (DORES; BENEVENUTO; LAENDER, 2017). 
Árvore de GA é uma estrutura em que cada vértice representa um orientador acadêmico e as arestas (direcionadas) representam as relações de orientação. A estruturação de árvores de genealogia acadêmica, por meio de relações de orientação, pode ser de grande utilidade para o registro histórico de grupos atuantes em específicas áreas do conhecimento (ROSSI; MENACHALCO, 2014).

Para Malmgren et al. (2010), a GA busca, por meio do registro de acadêmicos em diversas áreas e seus relacionamentos de orientação, documentar de forma perene a história de comunidades acadêmico-científicas e, assim, oferecer um melhor entendimento sobre como o passado impactou na formação do presente e qual a tendência para o futuro dessas comunidades. O objeto da pesquisa genealógica está, portanto, ligado à investigação dos ascendentes e descendentes de um indivíduo. A identificação dos graus de vínculos entre os indivíduos pode ser encontrada nos registros históricos, ou seja, em documentos que forneçam indícios de suas ligações (ROSSI; MENA-CHALCO, 2014).

Apesar de, às vezes, ser vista como uma forma de homenagear cientistas ou de saciar a curiosidade sobre os ancestrais de maior prestígio, a GA pode contribuir para um entendimento mais amplo sobre o papel do cientista na formação de novas gerações, a origem, desenvolvimento e desdobramentos de áreas do conhecimento (MEDEIROS; BARATA, 2017).

Além disso, Dores, Benevenuto, Laender (2017) afirmam que os estudos de GA, entre outras possibilidades, permitem: i) identificar os pesquisadores importantes dentro das áreas e o papel que desempenharam na criação e evolução de comunidades científicas e, até mesmo, de novas áreas; ii) compreender melhor o surgimento de pesquisas em determinadas áreas, o nascimento e a morte de comunidades de pesquisa; e, iii) identificar a linhagem acadêmica e o papel da formação interdisciplinar sobre a evolução de campos específicos de pesquisa.

A atividade de orientação e supervisão acadêmica é uma forma de relacionamento que promove a evolução do orientado, da instituição, da ciência e da sociedade de modo geral. Embora a vida do acadêmico seja finita, suas contribuições científicas são impactadas por meio de sucessivas gerações de 
orientados (SUGIMOTO, 2014; CASTANHA; GRÁCIO, 2015). Assim, os estudos de GA contribuem para o conhecimento sobre a herança intelectual de pesquisadores, operacionalizada pelas relações interdependentes entre os orientados e seus orientadores.

Conforme Mena-Chalco (2016), Rossi et al. (2018) e Sugimoto (2014), baseados nos distintos objetivos dos trabalhos de GA que vêm sendo desenvolvidos, os estudos genealógicos podem ser classificados em cinco tipos, conforme resumido a seguir.

- Honorífica - estudo da descendência de um indivíduo de interesse (ou grupo de indivíduos) com o objetivo de homenageálo por meio de sua linhagem, evidenciando sua relevância devido à contribuição exercida na formação da comunidade em função de suas orientações acadêmicas.

- Egocentrista - tipo de genealogia que utiliza a ascendência de um indivíduo de interesse a fim de identificar ancestrais ilustres e comprovar uma ligação, ainda que remota, entre ambos. Comumente a motivação para esse tipo de estudo está relacionada com curiosidade ou autopromoção.

- Histórica - utilizada como ferramenta complementar a estudos que visam a identificação de personagens históricos importantes para determinadas áreas do conhecimento. Permite contextualizar as relações entre os indivíduos identificados.

- Paradigmática - considerado como um dos tipos de GA mais inserido no contexto científico. Utiliza os relacionamentos orientador-orientado para estudar a extensão do conhecimento transmitido entre essas relações.

- Analítica - devido ao crescimento do número de bases de dados genealógicos e o desenvolvimento de análises estatísticas, a genealogia analítica surge como ferramenta para a avaliação e, 
por vezes, predição de padrões entre os membros de comunidades acadêmicas que são objeto de estudo.

Assim, considerando os seus objetivos, este trabalho pode ser enquadrado nos estudos de genealogia do tipo analítica, devido ao seu propósito de prover atributos quantitativos a relação de orientação acadêmica dos bolsistas PQ1-CNPq em CI como forma de mapear a propagação do conhecimento através do pesquisador orientador.

Os estudos genealógicos do tipo analítico utilizam dados científicos sobre os desdobramentos de pesquisas e das relações de orientações acadêmicas extraídos de bancos de dados que, geralmente, estão disponíveis em meios eletrônicos, com o propósito de, a partir de métricas estatísticas e de análises de redes sociais, descrever e medir práticas científicas (SILVA; NUNES; VIEGAS, 2018).

Nesse contexto, Rossi et al. (2018) descrevem um conjunto de métricas (que foram denominadas pelos autores de métricas genealógicas) que podem ser utilizadas para a caracterização de gráficos de GA. Uma métrica genealógica pode ser entendida como sendo um atributo quantitativo que define as características topológica da estrutura do gráfico genealógico e é semanticamente estável. Além disso, um gráfico genealógico tem características peculiares, que podem o diferenciar das redes sociais tradicionais. Desse modo, conforme os autores, o desenvolvimento de métricas específicas com a finalidade de caracterizar gráficos genealógicos contribui para a caracterização de indivíduos e grupos (ROSSI et al., 2018). Entre as métricas apresentadas por Rossi et al. (2018), escolheu-se três para serem analisadas neste estudo, são elas: fecundidade, descendência e geração ou linhagem acadêmica.

A fecundidade acadêmica é o número de filhos acadêmicos (descendentes diretos) que um acadêmico possui e refere-se ao número de orientados de um acadêmico. A métrica define a produtividade de um acadêmico e reflete a contribuição direta exercida na formação da comunidade, pelo número de pessoas treinadas por um determinado orientador (ROSSI et al., 2018). 
A descendência acadêmica de um pesquisador pode ser definida como sendo o conjunto de todos os indivíduos (pesquisadores) de uma árvore genealógica acadêmica que possuem um ancestral (orientador) em comum. Por descendentes, consideramos os filhos acadêmicos, netos acadêmicos, bisnetos acadêmicos e assim por diante, de um determinado pesquisador (ROSSI et al., 2018). Essa métrica é importante para verificar o impacto de um determinado acadêmico na formação da comunidade científica. Por outro lado, a relevância percebida de um acadêmico está sempre sujeita ao desempenho de seus filhos. Assim, o número de descendentes pode pressupor classificações imprecisas, quando consideradas individualmente (ROSSI et al., 2018).

A linhagem acadêmica de um pesquisador é igual ao número de gerações de orientados presentes na árvore genealógica dele. Os descendentes diretos, ou seja, os filhos acadêmicos, constituem a primeira geração, os netos representam a segunda geração, os bisnetos a terceira geração, mantendo esse padrão continuamente. O número de gerações de um acadêmico é um indicativo do impacto, perpetuação e evolução de suas ideias e conhecimentos na comunidade em que está inserido (ROSSI et al., 2018).

\section{Procedimentos metodológicos}

No presente trabalho, foi utilizada como principal fonte de dados os currículos da Plataforma Lattes (CV Lattes). A Plataforma Lattes representa a experiência do CNPq na integração de bases de dados de Currículos, de Grupos de pesquisa e de Instituições em um único Sistema de Informações, se tornando um padrão nacional no registro da vida pregressa e atual de estudantes e pesquisadores do país.

Para Lane (2010), a Plataforma Lattes apresenta-se como o maior e mais relevante repositório curricular de acadêmicos relacionados com a ciência brasileira. Essa plataforma é uma iniciativa ímpar em registrar as atividades de milhões de acadêmicos. Por meio de currículos acadêmicos, ela disponibiliza informações sobre formação, produção científica, participação em eventos, dentre outros registros acadêmicos relevantes. 
No entanto, Rossi, Damaceno e Mena-Chalco (2018) alertam para algumas limitações da Plataforma Lattes no tocante às informações acadêmicogenealógicas, na quais são descritas os ascendentes e descendentes do acadêmico. Os registros de orientações dos CV Lattes possuem a opção de vincular manualmente o nome do orientado ao seu identificador único na Plataforma Lattes (ID Lattes). No entanto, em geral, relacionamentos antigos permaneceram sem seus vínculos com os identificadores, exibindo apenas o nome do registro de orientação. Dessa forma, as análises das orientações acadêmicas ficam prejudicadas.

Todavia, com o propósito de contribuir para a solução desse problema, Moreira et. al. (2018) desenvolveram um método para a caracterização de vínculos de orientações acadêmicas em grandes repositórios de dados ambíguos, como é o caso da Plataforma Lattes. Os autores afirmam que com a aplicação do método na extração de dados de orientações acadêmicas na Plataforma Lattes, foram identificados cerca de $38 \%$ mais relacionamentos que antes do processo de desambiguação (MOREIRA et al., 2018).

Entretanto, para este artigo, em função do tempo limitado, não foram aplicadas estratégias para a identificação dos relacionamentos de orientação acadêmica que porventura não tenham sido registrados no momento do cadastro dos dados de orientação nos CV Lattes.

\subsection{Coleta e análise dos dados}

Para a coleta e análise dos dados deste estudo foram adotadas as três etapas apresentadas a seguir.

A etapa 1 consistiu na identificação dos bolsistas, realizada a partir da página eletrônica de bolsas e auxílios vigentes do $\mathrm{CNPq}$ (<http://www.cnpq.br/web/guest/bolsistas-vigentes/>), deu-se com a busca: País (Brasil) AND Estado (Todos os Estados) AND Instituições (Todas as Instituições) $A N D$ Grandes Áreas (Ciências Sociais Aplicadas) AND Áreas (Ciência da Informação) $A N D$ Modalidades (Produtividade em Pesquisa) AND 
Níveis (Todos os Níveis). Com isso, identificou-se um universo composto por 16 bolsistas PQ1-CNPq e 37 bolsistas PQ2-CNPq, totalizando 53 bolsistas. Para este trabalho foram selecionados apenas os 16 bolsistas (PQ1-CNPq).

Para atender aos propósitos deste artigo foi realizado um novo levantamento de dados na página do CNPq no dia 28 de agosto de 2018. O quantitativo de pesquisadores PQ1-CNPq em CI com bolsas em vigência nessa data foi diferente dos dados coletados para a elaboração do trabalho que foi apresentado no $6^{\circ} \mathrm{EBBC}$.

Na etapa 2 foi realizada a extração de dados da Plataforma Lattes, com o objetivo de obter informações acadêmico-genealógicas sobre os bolsistas. Para isso, foi utilizado o LattesDataXplorer, proposto e desenvolvido por Dias (2016). Os dados extraídos foram enviados para o Gephi, software de análise de redes socais. Esse procedimento foi utilizado para gerar as árvores genealógicas acadêmica dos pesquisadores. Utilizou-se ainda a Plataforma Acácia ${ }^{2}$ para obter os dados dos bolsistas no tocante à ancestralidade e descendência acadêmica. Esses dados foram coletados manualmente.

$\mathrm{Na}$ etapa 3, os dados coletados na Plataforma Acácia, referente aos ascendentes (orientadores) e descendentes (orientados), foram organizados em planilhas Excel, gerando tabelas e gráficos. Com o Gephi foi possível construir as árvores genealógicas, facilitando a visualização dos dados. Para as análises foram consideradas as seguintes variáveis: fecundidade, descendência e geração ou linhagem acadêmica dos pesquisadores, conforme abordado no tópico 2 .

\section{Resultados}

Os resultados foram estruturados na seguinte ordem: (i) dados gerais dos pesquisadores, tais como filiação e ano de doutoramento, (ii) análises da fecundidade, descendência, (iii) linhagem ou geração acadêmica e (iv) ancestralidade dos bolsistas PQ1-CNPq em CI. 


\subsection{Dados gerais dos pesquisadores}

Foram extraídos da Plataforma Lattes os dados referentes à identificação, endereço, orientações e supervisões concluídas dos 16 bolsistas PQ1-CNPq em CI. A partir dos dados das orientações desses bolsistas foi possível identificar a sua ascendência e descendência acadêmica e, de certa maneira, verificar como ocorre a propagação do conhecimento a partir da relação orientador-orientado.

Foram identificados os 16 bolsistas, a filiação deles e o ano de doutoramento. Quanto às instituições de filiação dos bolsistas, o Instituto Brasileiro de Informação em Ciência e Tecnologia (IBICT) detém 1/4 dos pesquisadores, ou seja, quatro. Depois vem a Universidade de São Paulo (USP) com três pesquisadores. Na sequência a Universidade Estadual Paulista (Unesp), Universidade Federal de Pernambuco (UFPE), Universidade Federal Fluminense (UFF) e Universidade Federal do Estado do Rio de Janeiro (UNIRIO) com dois e Universidade de Brasília (UnB) com um pesquisador. Do total de bolsistas, dois concluíram o doutoramento no ano de 1989, sete cursaram entre os anos de 1991 a 1994 e outros sete concluíram o curso entre os anos de 1995 a 1999 (ver Quadro 1).

Nesse aspecto, não foi observado a influência do ano de doutoramento no desempenho dos pesquisadores, a princípio pensou-se que aqueles que se doutoraram primeiro poderiam ter melhor desempenho quanto ao número de orientados e de descendentes. Foi possível notar a existência de pesquisadores com desempenho superior independente do ano de doutoramento, por exemplo pesquisador NP 8 e NP 15.

Quadro 1 - Caracterização dos pesquisadores bolsistas do CNPq da área de CI por instituição e ano de doutoramento

\begin{tabular}{|c|l|c|c|}
\hline NP & \multicolumn{1}{|c|}{ NOME } & INS & ANO DOU \\
\hline 1 & Carlos Henrique Marcondes de Almeida & UFF & 1998 \\
\hline 2 & Emir José Suaiden & UnB & 1989 \\
\hline 3 & Gilda Olinto de Oliveira & IBICT & 1994 \\
\hline 4 & Icleia Thiesen & UNIRIO & 1997 \\
\hline 5 & José Augusto Chaves Guimarães & UNESP & 1993 \\
\hline 6 & José Maria Jardim & UNIRIO & 1998 \\
\hline 7 & Leilah Santiago Bufrem & UFPE & 1991 \\
\hline 8 & Lena Vania Ribeiro Pinheiro & IBICT & 1997 \\
\hline 9 & Maria de Fátima Gonçalves Moreira Tálamo & USP & 1989 \\
\hline
\end{tabular}




\begin{tabular}{|c|l|c|c|}
\hline 10 & Maria Nélida Gónzalez de Gómez & UFF & 1992 \\
\hline 11 & Mariângela Spotti Lopes Fujita & UNESP & 1992 \\
\hline 12 & Marilda Lopes Ginez de Lara & USP & 1999 \\
\hline 13 & Nair Yumiko Kobashi & USP & 1994 \\
\hline 14 & Raimundo Nonato Macedo dos Santos & UFPE & 1995 \\
\hline 15 & Regina Maria Marteleto & IBICT & 1992 \\
\hline 16 & Sarita Albagli & IBICT & 1997 \\
\hline
\end{tabular}

Legenda: NP - Número do pesquisador / INS - Instituição / DOU - Doutoramento. Fonte: Plataforma Acácia (2018).

\subsection{Fecundidade e descendência acadêmica}

Os bolsistas orientaram 627 pesquisadores ao longo da sua trajetória acadêmica, em média cada bolsista orientou 39,19 pesquisadores, com uma mediana igual a 36. Com relação ao número de descendentes, os bolsistas tiveram 1.075 descendentes no total, com média de 67,19 pesquisadores por bolsista, com mediana igual 48. Apresenta-se a seguir (ver Tabela 2) a taxa de fecundidade, ou seja, número de indivíduos orientados por um acadêmico durante a sua carreira e a taxa de descendentes, ou seja, o conjunto de todos os pesquisadores da árvore genealógica que tem em comum o mesmo orientador. Como pode ser observado, os três primeiros bolsistas (PQ1-CNPq) estão entre os melhores ranqueados, a bolsista Lena Vania Ribeiro Pinheiro merece destaque, orientando 94 pesquisadores, $14,99 \%$ do total. Seguida por Icleia Thiesen e Maria Nélida Gónzalez de Gómez com, respectivamente, 55 e 51 pesquisadores orientados.

Em relação à descendência, Maria Nélida Gónzalez de Gómez é a bolsista com maior número de descendentes, 226 no total, contando filhos, netos, bisnetos e tataranetos. Na sequência, estão Regina Maria Marteleto e Lena Vania Ribeiro Pinheiro com, respectivamente, 189 e 148 descendentes. Foi possível verificar que maior fecundidade não implica, necessariamente, em maior número de descendentes, pois a descendência depende da permanência dos orientados no meio acadêmico orientando outros pesquisadores.

Tabela 1 - Taxa de fecundidade e descendência dos bolsistas PQ1-CNPq da área de CI

\begin{tabular}{|l|c|c|c|c|}
\hline \multicolumn{1}{|c|}{ NOME } & FEC & $\%$ & DES & $\%$ \\
\hline Lena Vania Ribeiro Pinheiro & 94 & 14,99 & 148 & 13,77 \\
\hline Icleia Thiesen & 55 & 8,77 & 45 & 4,19 \\
\hline Maria Nélida Gónzalez de Gómez & 51 & 8,13 & 226 & 21,02 \\
\hline
\end{tabular}




\begin{tabular}{|l|c|c|c|c|}
\hline Raimundo Nonato Macedo dos Santos & 50 & 7,97 & 59 & 5,49 \\
\hline Regina Maria Marteleto & 48 & 7,66 & 189 & 17,58 \\
\hline José Augusto Chaves Guimarães & 46 & 7,34 & 22 & 2,05 \\
\hline Gilda Olinto de Oliveira & 37 & 5,9 & 19 & 1,77 \\
\hline Leilah Santiago Bufrem & 37 & 5,9 & 66 & 6,14 \\
\hline Emir José Suaiden & 35 & 5,58 & 52 & 4,84 \\
\hline $\begin{array}{l}\text { Maria de Fátima Gonçalves Moreira } \\
\text { Tálamo }\end{array}$ & 31 & 4,94 & 57 & 5,3 \\
\hline Nair Yumiko Kobashi & 30 & 4,78 & 51 & 4,74 \\
\hline Mariângela Spotti Lopes Fujita & 28 & 4,47 & 45 & 4,19 \\
\hline José Maria Jardim & 27 & 4,31 & 16 & 1,49 \\
\hline Sarita Albagli & 25 & 3,99 & 30 & 2,79 \\
\hline Carlos Henrique Marcondes de Almeida & 17 & 2,71 & 21 & 1,95 \\
\hline Marilda Lopes Ginez de Lara & 16 & 2,55 & 29 & 2,7 \\
\hline
\end{tabular}

Legenda: FEC - Fecundidade / DES - Descendência.

Fonte: Plataforma Acácia (2018).

Uma outra análise realizada foi com relação a floresta genealógica dos bolsistas de produtividade. Uma floresta é caracterizada com a inserção de todas as árvores genealógicas que foram caracterizadas, formando um conjunto de árvores e suas possíveis ligações (ver Figura 1).

Como pode ser verificado, ao realizar a inserção das árvores de cada um dos bolsistas PQ1-CNPq analisados, formando uma floresta de árvores, algumas árvores estão conectadas por alguns elementos. A componente gigante, que se caracteriza pelo componente com o maior número de nós, engloba 14 dos 16 bolsistas, ou seja, apenas duas bolsistas estão isoladas na floresta, ou seja, não possuem ligações com nenhuma das outras árvores.

Ao analisar os vínculos de orientações entre diferentes árvores, foi possível perceber que em geral são de uma mesma instituição, e que um determinado bolsista orientou um aluno em seu mestrado e outro bolsista orientou esse mesmo aluno em seu doutorado, caracterizando um vínculo de orientação; sendo esse aluno orientado por dois bolsistas distintos o responsável por estar conectando as duas árvores. Situação que ocorreu em diversas ocasiões. 


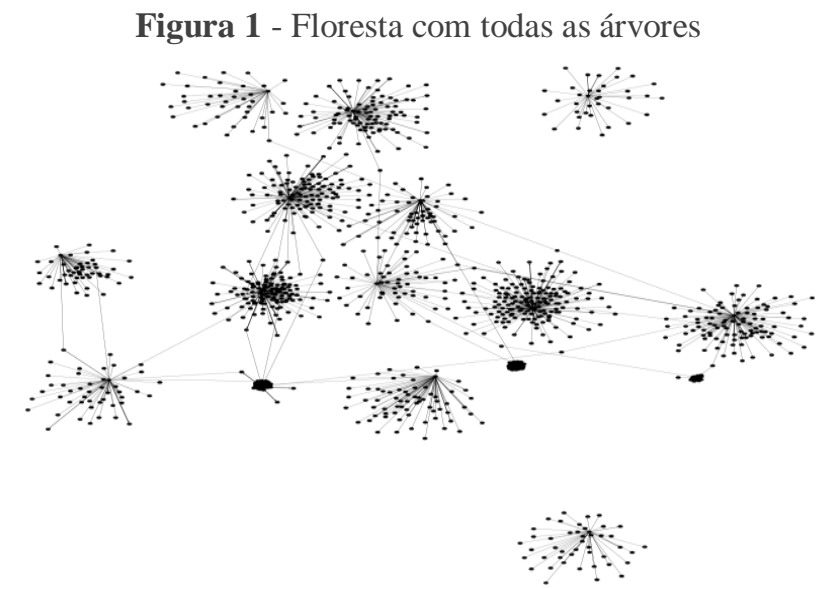

Fonte: Elaborado pelos autores (2018).

Além disso, também foi possível identificar uma determinada bolsista (Icleia Thiesen) que foi orientada por outra bolsista (Maria Nélida Gónzalez de Gómez), interligando as duas árvores, e ainda, um bolsista (José Maria Jardim) que teve orientação de outros dois bolsistas (Regina Maria Marteleto e Maria Nélida Gónzalez de Gómez), tornando as suas árvores também interconectadas.

\subsection{Linhagem ou geração acadêmica}

Com relação à linhagem ou geração acadêmica dos bolsistas, ou seja, o número de gerações de pesquisadores que foram influenciadas pelos bolsistas, observouse que Maria Nélida Gónzalez de Gómez possui a árvore genealógica com a maior linhagem ou geração acadêmica, tendo influenciado quatro gerações de pesquisadores.

Com relação aos demais bolsistas, observou-se que a linhagem ou geração acadêmica de bolsistas corresponde a três gerações de pesquisadores e outros três bolsistas influenciaram duas gerações de pesquisadores. As árvores genealógicas dos bolsistas: Maria Nélida Gónzalez de Gómez (quatro gerações), Lena Vania Ribeiro Pinheiro (três gerações) e José Maria Jardim (duas gerações), respectivamente, são apresentadas na sequência. (ver Figura 2). 
Figura 2 - Linhagem ou geração acadêmica dos bolsistas PQ1-CNPq em CI
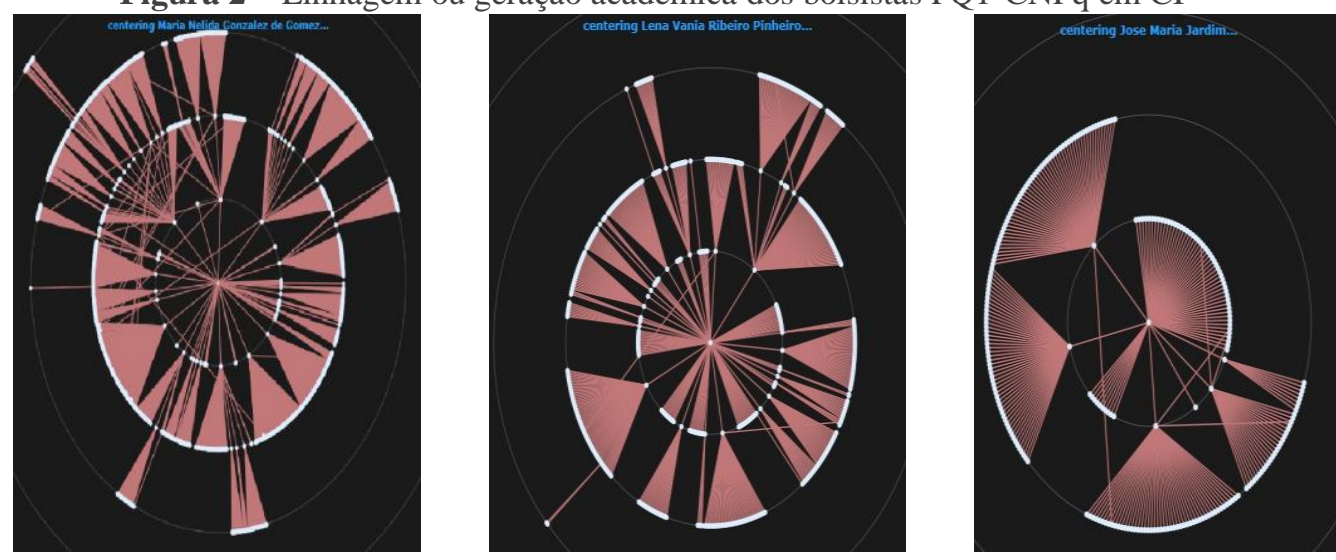

Fonte: Elaborado pelos autores (2018).

Observa-se que o bolsista pesquisado está no centro de cada árvore genealógica, ele é o pai genealógico ou ancestral raiz, sendo que cada circunferência representa uma nova geração de orientados. Nas árvores genealógicas apresentadas na Figura 2 é possível notar que a primeira alcançou quatro gerações de pesquisadores, enquanto a segunda alcançou três e a terceira alcançou duas, tendo uma linhagem acadêmica mais baixa.

\subsection{Ascendência acadêmica}

Um último aspecto analisado foi a ascendência dos bolsistas. Verificou-se até a quarta geração de antecedentes dos pesquisadores com o objetivo de identificar ancestrais acadêmicos brasileiros com significativa participação na formação de pesquisadores na CI, além de verificar entre os ancestrais estrangeiros aqueles que mais contribuíram para a formação de pesquisadores brasileiros da área. Para todos os bolsistas, o ancestral raiz foi considerado um pesquisador estrangeiro, independentemente da geração que se encontrava, pois a Plataforma Lattes não contempla o currículo desses pesquisadores.

Desse modo, identificou-se 41 ancestrais acadêmicos orientador ou coorientador de mestrado e/ou doutorado, sendo 22 pesquisadores nacionais e/ou vinculados a instituições brasileiras e 19 pesquisadores estrangeiros. Todos os pesquisadores estrangeiros da árvore genealógica de ancestralidade orientaram apenas um pesquisador brasileiro, seja no mestrado ou doutorado. 
Não havendo assim entre os ancestrais acadêmicos estrangeiros dos bolsistas um orientador que fosse o ancestral raiz.

Entre os ancestrais nacionais dos bolsistas que mais contribuíram na orientação de pesquisadores estão: Aldo de Albuquerque Barreto (quatro orientações); Johanna Wilhelmina Smit (quatroorientações); Gilda Maria Braga (três orientações); Frederic M. Litto (três orientações); Maria Nélida Gónzalez de Gómez (duas orientações) e José Teixeira Coelho Netto (duas orientações), os demais contribuíram com uma orientação cada. Ressalta-se que Aldo de Albuquerque Barreto foi orientador da bolsista Maria Nélida Gónzalez de Gómez no doutorado, essa, por sua vez, orientou outros dois bolsistas (Icleia Thiesen e José Maria Jardim).

\section{Considerações finais}

O estudo obteve resultados que permitem olhar de forma complementar aos habituais estudos quantitativos de produção da comunidade científica, apesar da limitação imposta pela Plataforma Lattes na extração de todos os dados de orientação.

Nota-se que a CI brasileira atual está representada por 15 mestrados acadêmicos, oito mestrados profissionais e 11 doutorados. Esse conjunto é ofertado por 16 universidades federais e quatro universidades estaduais. O que tem contribuído significativamente para a capacitação de profissionais que têm atuado na CI em diversos segmentos (CAPES, 2018). Mas, a área detém, nesse momento, apenas 16 bolsistas, número relativamente baixo diante da expansão do número de programas de pós-graduação. Todavia, há outras questões que influenciam o número de bolsistas por área de conhecimento, mas que não foram exploradas por não serem objetivo deste trabalho.

Além disso, um dos resultados relevantes foi esclarecer que a taxa de fecundidade maior dos bolsistas não significa necessariamente uma descendência extensa de professoreslpesquisadores. A descendência é importante, neste estudo, quando continua em atividades acadêmicas. Outro 
resultado que chamou a atenção foi a existência entre os bolsistas PQ1-CNPq de pesquisadores que foram orientadores no mestrado e/ou doutorado de outros bolsistas, demonstrando o mérito da orientação acadêmica na formação de novos pesquisadores para a área da CI brasileira.

Além dos aspectos já mencionados, este estudo possibilitou reconhecer também, pela abordagem da formação de novos pesquisadores para CI, as notáveis contribuições de pesquisadores que já são reconhecidos na área devido as suas produções científicas.

Dois aspectos foram limitantes para a realização deste estudo. Primeiro, conforme foi detalhado nos procedimentos metodológicos, a utilização dos dados da Plataforma Lattes não é trivial, seja pela ineficiência do seu sistema de buscas e recuperação de dados, que depende que o pesquisador preencha o seu CV Lattes, gerando uma desatualização e confiabilidade questionável, ou mesmo pela própria manipulação dos dados que exige conhecimentos e técnicas que fogem do usuário comum.

Soma-se a isso as restrições de acesso impostas pelo CNPq, sendo que somente usuários autorizados podem ter acesso mais completo a dados de conjuntos de currículos. Dessa forma, é possível que outros dados referentes à orientação e supervisão acadêmica dos bolsistas PQ1-CNPq não tenham sido identificados.

Segundo, com relação ao grupo de bolsistas estudado, salienta-se que embora eles possuam notória atuação em suas áreas, eles não são os únicos a desenvolver pesquisas na CI. Reconhece-se a existência de outros importantes pesquisadores da área que, por razões diversas, desde aposentaria ou mesmo desinteresse em participar do processo de seleção para bolsistas, não são contemplados pela bolsa de produtividade e, consequentemente, não estão incluídos no escopo deste estudo.

Para estudos futuros, sugere-se replicar este trabalho com todos os bolsistas $\mathrm{CNPq}$ da área de CI, explorando métricas que não foram analisadas neste artigo. 


\section{Referências}

BIANCHETTI, Lucídio; MACHADO, Ana Maria Netto (Org.). A bússola do escrever: desafios e estratégias na orientação e escrita. 3. ed. São Paulo: Cortez, 2012. $412 \mathrm{p}$.

BUFREM, Leilah Santigo; SILVA, Fábio Mascarenhas; SOBRAL, Natanael Vitor. Análise das influências intelectuais na produção científica da área de Ciência da Informação: um estudo sobre os bolsistas de produtividade em pesquisa (PQ-CNPq). Em Questão, v. 23, p.115-141, jan. 2017. http://dx.doi.org/10.19132/1808-5245230.115-141

CAFÉ, Anderson. et al. A Elite Acadêmica da Sociologia no Brasil e sua Produção Científica. Informação \& Informação, v. 16, n. 3, p. 19-39, maio 2012. Universidade Estadual de Londrina. http://dx.doi.org/10.5433/19818920.2011v16n3p19

CASTANHA, Renata Cristina Gutierres; GRÁCIO, Maria Claúdia Cabrini Estudos de genealogia acadêmica como abordagem para análise de domínio. 2015. Disponível em: https://www.researchgate.net/profile/Renata_Castanha/publication/280529962 Academic_Genealogy_studies_as_approach_for_Domain_Analysis/links/5609af 7608ae576ce63e7960/Academic-Genealogy-studies-as-approach-for-DomainAnalysis.pdf. Acesso em: 14 dez. 2018.

CORDOVA, Kyle. E.; FURUKAWA, Hiroyasu; YAGHI, Omar M. The Development of Global Science. Acs Central Science, v. 1, n. 1, p.18-23, 23 mar. 2015. American Chemical Society (ACS). http://dx.doi.org/10.1021/acscentsci.5b00028

DORES, Wellington; BENEVENUTO, Fabrício; LAENDER, Alberto, H. F. Building the Brazilian Academic Genealogy Tree. In: KAMPS, J.; TSAKONAS, G.; MANOLOPOULOS, Y.; ILIADIS, L.; KARYDIS I. (Ed.). Research and Advanced Technology for Digital Libraries. TPDL 2017. Lecture Notes in Computer Science, v. 10450. Springer, Cham

OLIVEIRA, Carlos Alexandre de; COSTA, Belkiz Inez Rezende; OLIVEIRA, Marlene. Genealogia acadêmica: um estudo dos pesquisadores da área de Ciência da Informação com bolsas produtividade em pesquisa na modalidade PQ-1 do CNPq. In: ENCONTRO BRASILEIRO DE BIBLIOMETRIA E CIENTOMETRIA, 6., 2018, Rio de Janeiro. Anais... Rio de Janeiro: UFRJ, 2018. p. 291 - 300. Disponível em: https://ebbc.inf.br/ebbc6/index.php/anais. Acesso em: 09 fev. 2018.

GUEDES, Vânia L. S.; BORSCHIVER, Suzana. Bibliometria: uma ferramenta Estatística para a gestão da informação e do conhecimento, em sistemas de Informação, de comunicação e de avaliação científica e tecnológica. In: ENCONTRO NACIONAL DE CIÊNCIA DA INFORMAÇÃO, 6., 2005, 
Salvador. Anais... Salvador: ICI/UFBA, 2005. Disponível em: < http://www.cinform-anteriores.ufba.br/vi_anais/docs/VaniaLSGuedes.pdf >. Acesso em: 23 set. 2018.

GUIMARÃES, José Augusto Chaves; GRÁCIO, Maria. Claúdia Cabrini; MATOS, Daniela Fernada de Oliveira. Produção científica de bolsistas pesquisa em Ciência da Informação do Conselho Nacional de Desenvolvimento Científico e Tecnológico (CNPq) - um estudo com artigos de periódicos. Datagramazero, v. 15, n. 2, abr. 2014. Disponível em: < http://hdl.handle.net/11449/114848 f>. Acesso em: 23 set. 2018.

HEINISCH, Dominik P.; BUENSTORF, Guido. The next generation (plus one): an analysis of doctoral students' academic fecundity based on a novel approach to advisor identification. Scientometrics, v. 117, n. 1, p.351-380, jul. 2018. http://dx.doi.org/10.1007/s11192-018-2840-5.

LANE, Julia Let's make Science metrics more scientific. Nature, v. 464, n. 7388, p. 488, 2010. Disponível em: <https://www.nature.com/articles/464488a〉. Acesso em: 24 set. 2018.

Le COADIC, Yves François. A Ciência da Informação. Brasília: Briquet de Lemos, 1996.

MALMGREN, R. Dean; OTTINO, Julio M.; AMARAL, Luís A. Nunes. The role of mentorship in protégé performance. Nature, v. 465, n. 7298, p.622-626, jun. 2010. Springer Nature. http://dx.doi.org/10.1038/nature09040.

MEADOWS, Arthur Jack. A comunicação científica. Brasília: Briquet de Livros 1999.

MEDEIROS, Carolina; BARATA, Germana. Perspectivas para a história e para a produção da ciência. Cienc. Cult., v. 69, n. 1, p. 22-24, mar. 2017. http://dx.doi.org/10.21800/2317-66602017000100011.

MENA-CHALCO, Jesús P. Genealogia acadêmica: uma ferramenta para investigar a origem, evolução e disseminação de áreas do conhecimento. In: ENCONTRO BRASILEIRO DE BIBLIOMETRIA E CIENTOMETRIA, 5., 2016, São Paulo. [Workshop]. São Paulo: ECA/USP, 2016. Disponível em: https://www.researchgate.net/publication/305082103_Genealogia_academica_u ma_ferramenta_para_investigar_a_origem_evolucao_e_disseminacao_de_areas _do_conhecimento?channel=doi\&linkId=5781059608ae5f367d392783\&showF ulltext=true. Acesso em: 24 set. 2018.

MENA-CHALCO, Jesús P. et al. Brazilian bibliometric coauthorship networks. Journal of The Association For Information Science And Technology, v. 65, n. 7, p.1424-1445, jan. 2014. Wiley. http://dx.doi.org/10.1002/asi.23010 
MOREIRA, Tales Henrique José et. al. Árvores genealógicas acadêmicas como estratégia para análises de processo de orientação. Pesq. Bras. em Ci. da Inf. e Bib., v. 13, n. 2, p. 246-254, 2018. https://doi.org/10.22478/ufpb.1981$0695.2018 \mathrm{v} 13 \mathrm{n} 2.42880$

PLATAFORMA ACACIA. A genealogia acadêmica do Brasil. 2018. Disponível em: http://plataforma-acacia.org/. Acesso em: 24 set. 2018.

ROSSI, Luciano; DAMACENO, Rafael Jeferson Pezzuto; MENA-CHALCO, Jesús P. Genealogia acadêmica: um novo olhar sobre impacto acadêmico de pesquisadores. In: SEMINÁRIO DE AVALIAÇÃO DE POLÍTICAS DE CT\&I, 1., 2018. Anais... Brasília, 2018. Disponível em: https://www.researchgate.net/publication/326960663_Genealogia_academica_U m_novo_olhar_sobre_impacto_academico_de_pesquisadores. Acesso em: 24 set. 2018.

ROSSI, Luciano; MENA-CHALCO, Jesús P. Caracterização de árvores de genealogia acadêmica por meio de métricas em grafos. In: CONGRESSO DA SOCIEDADE BRASILEIRA DE COMPUTAÇÃO, 34., 2014, Brasília. Anais... Brasília: SBC, 2014. p. 21-32. Disponível em:

http://each.uspnet.usp.br/digiampietri/BraSNAM/2014/p02.pdf . Acesso em: 24 set. 2018.

ROSSI, Luciano. et al. Topological metrics in academic genealogy graphs. Journal of Informetrics, v. 12, n. 4, p. 1042-1058, 2018. Disponível em:

https://reader.elsevier.com/reader/sd/pii/S1751157718300750?token=DD2D262 0F4F0CE95FCAC6DB1B9EB804C1AB076FB7707DD223E0B10C081632B63 BB998728DC79655782721E29CB43620F . Acesso em: 24 set. 2018.

SILVA, Carlos Eduardo M. Viegas; NUNES, Rubens.; VIEGAS, Elisabete Maria Macedo A genealogy of the Brazilian scientific research on freshwater fish farming by means of the academic supervision linkage. Scientometrics, v. v. 117, n. 3, p.1535-1553, 2018. https://doi.org/10.1007/s11192-018-2940-2

SUGIMOTO, Cassidy R. Academic genealogy. In: CRONIN, Blaise; SUGIMOTO, Cassidy R. (Ed.). Beyond bibliometrics: harnessing multidimensional indicators of scholarly impact. Cambridge, Massachusetts: MIT Press, 2014. p. 365-382. 


\title{
Academic genealogy in the field of Information Science: a study on researchers with productivity grants (PQ-CNPq)
}

\begin{abstract}
The article aims to investigate the academic genealogy of the scholarship researchers of research productivity from the National Council for Scientific and Technological Development, level 1, (PQ1-CNPq) on Library and Information Science (LIS). Thereby, it is expected mapping the knowledge propagation through the advisor researcher, also evidencing the contributions of researchers in the education of Human Resources for the field. Methodological proceedings are: (1) identification of the scholarship researchers of productivity on LIS research; (2) data collection at Lattes and Acácia platforms; (3) analysis and presentation of the researchers' academic genealogical trees. The results show that the PQ1-CNPq on LIS advised 627 researchers, obtained 1.075 descendants and a fourth generation academic lineage. The academic ascendants of the scholarship researchers were also identified, highlighting those academic ancestors that contributed the most for the education of generations of researchers on Brazilian LIS.
\end{abstract}

Keywords: Academic genealogy. Academic advising. Library and Information Science (LIS). Scholarship of research productivity. Genealogical studies.

Recebido: $24 / 09 / 2018$

Aceito: 03/12/2018

\footnotetext{
${ }^{1} \mathrm{Na}$ sequência do artigo será adotado o termo "orientação acadêmica" por entender que é mais comum no meio acadêmico brasileiro.

${ }^{2}$ A Plataforma Acácia é uma iniciativa de um grupo de pesquisadores brasileiros desenvolvida com o objetivo de fornecer informações acadêmico-genealógicas sobre a comunidade acadêmico-científica no Brasil. Pode ser acessada no seguinte endereço eletrônico: <http://plataforma-acacia.org/>.
} 